\title{
Quantidade de geração de resíduos em seis procedimentos cirúrgicos de portes diferenciados comparados
}

\author{
Quantity of generation of waste in six surgical of differents ports procedures comparative \\ Roberto Naime'; Cristiane Harutunian Barbisan² \\ 'Professor do Programa de pós-graduação em Qualidade Ambiental - Universidade FEEVALE \\ ${ }^{2}$ Acadêmica de Ciências Biológicas - Universidade FEEVALE
}

\begin{abstract}
Resumo
Os riscos à saúde e ao meio ambiente provocados por resíduos sólidos nos serviços da saúde (RSSS) são devido principalmente às suas frações infectantes. Esses resíduos apresentam microrganismos que são patogênicos e apresentam riscos para a saúde da população e para o meio ambiente. Existe a falta de orientação sobre os riscos potenciais, manuseio e tratamento, assim como a ausência de informações quanto à forma de tratamento e à destinação final destes resíduos. Este trabalho faz um levantamento sobre a natureza e quantidade de resíduos gerados em dois procedimentos cirúrgicos de diferentes portes em um hospital de Porto Alegre que é reconhecidamente gerador de grande quantidade de resíduos. O estudo foi patrocinado pelo hospital que busca através deste conhecimento estabelecer normas e procedimentos adequados para atender satisfatoriamente as demandas de gestão de resíduos sólidos hospitalares. Foram comparados seis procedimentos cirúrgicos de grande freqüência no hospital, que são a colicistectomia e a revascularização do miocárdio, objetivando mensurar a quantidade de resíduos biológicos, recicláveis, não-recicláveis e secreções produzidas de cada um deles, visando estabelecer rotinas mais adequadas para o gerenciamento dos resíduos em condições de proteção ambiental adequadas e com o menor custo possível. Foram realizadas pesagens de cada um dos tipos de resíduos gerados nas cirúrgias e feitas análises estatísticas para validação dos resultados. O trabalho demonstra que a natureza do procedimento, quando comparado, influencia na quantidade e tipologia da geração de resíduos numa intervenção cirúrgica. Palavras Chave: resíduos de saúde, gravimetria, destinação
\end{abstract}

\begin{abstract}
The risks to health and the environment caused by solid waste in health services (SRHS) is mainly due to their infectious fractions. These wastes have microorganisms that are pathogenic and pose a risk to public health and the environment. There is a lack of guidance on the potential risks, handling and treatment, as well as the lack of information on how the treatment and disposal of these wastes. This work is a survey on the nature and amount of waste generated in two surgical procedures of different sizes in a hospital in Porto Alegre, which is known to generate large quantities of waste. The study was sponsored by the hospital to search through this knowledge to establish standards and procedures to satisfactorily meet the demands of managing solid waste. We compared six surgical procedures that happen frequently in the hospital, aiming to measure the amount of organic waste, recyclable, non-recyclable and secretions produced in each of them, to establish routines for the most appropriate management of waste under conditions of environmental protection and appropriate the lowest possible
\end{abstract}




\section{INTRODUÇÃO}

Preservar o meio ambiente é uma necessidade universal para a preservação de nossa espécie. E preservar a espécie humana não teria sentido sem padrões mínimos e dignos de bem-estar. Toda e qualquer obra ou tarefa que persiga esses dois objetivos será uma importante contribuição para uma missão que é de todos nós (MAGALHÃES, 1995).

A preocupação com o meio ambiente aumenta a medida em que ocorre o crescimento populacional e a industrialização. Nacionalmente, um dos principais assuntos discutidos é a reciclagem dos resíduos sólidos gerados por mais de 180 milhões de brasileiros (IBGE, 2008). Há uma parte que são resíduos recicláveis e devem ter esta destinação. Mas há uma parte que deve receber tratamento adequado com a destinação para Aterros de Resíduos Industriais Perigosos (ARIPs) ou tratamento prévio com autoclavagem e destinação final em ARIPs.

Rebelatto (2006), citando dados da Pesquisa Nacional de Saneamento Básico, realizada pela Fundação Instituto Brasileiro de Geografia e Estatística (IBGE) em 2000, aponta que 74\% dos municípios brasileiros depositam "lixo hospitalar" a céu aberto, $57 \%$ separam os dejetos nos hospitais e apenas $14 \%$ das prefeituras tratam adequadamente os resíduos de serviços de saúde. Em 2000, a situação de disposição e tratamento dos Resíduos Sólidos de Serviços de Saúde - RSSS - melhorou com relação aos dados de 1989, com 9,5\% dos municípios encaminhando-os para aterros de resíduos especiais (69,9\% próprios e $30,1 \%$ de terceiros). Em número de municípios, 2.569 depositam-nos nos mesmos aterros que os dos resíduos comuns, enquanto 539 já estão enviando-os para locais de tratamento ou aterros de segurança.

A crescente consciência sobre os riscos à saúde pública e ao meio ambiente, provocados por RSSS, deve-se, principalmente, as suas frações infectantes. No Brasil há mais de 30 mil unidades de saúde produzindo esses resíduos, e na maioria das cidades, a questão do manuseio e da disposição final não está resolvida, e acrescenta-se que algumas unidades de saúde desconhecem a quantidade e a composição dos resíduos que produzem (FERREIRA, 1995).

A Resolução da Diretoria Colegiada da ANVISA - RDC 306/ 2004 classifica os Resíduos dos Serviços de Saúde em cinco grupos:
- GRUPO A - resíduos com risco biológico: resíduos com possível presença de agentes biológicos que, por suas características de maior virulência ou concentração, podem apresentar risco de infecção.

- GRUPO B - resíduos com risco químico: resíduos contendo substâncias químicas que podem apresentar risco à saúde pública ou ao meio ambiente, dependendo de suas características de inflamabilidade, corrosividade, reatividade e toxicidade.

- GRUPO C - rejeitos radioativos: rejeitos de qualquer material resultante de atividades humanas que contenham radionuclídeos em quantidades superiores aos limites de isenção especificados na norma CNEN-6.02, e para as quais a reutilização é imprópria ou não prevista.

- GRUPO D - resíduos comuns: todos os resíduos gerados nos serviços abrangidos pela resolução RDC 306 da ANVISA, que por suas características, não necessitam de processos diferenciados relacionados ao acondicionamento, identificação e tratamento, devendo ser considerados resíduos sólidos urbanos - RSU. Podem ser classificados em recicláveis e não recicláveis.

- GRUPO E - perfurocortantes: objetos e instrumentos contendo cantos, bordas, pontos ou protuberâncias rígidas e agudas, capazes de cortar ou perfurar. Neste podemos encontrar lâminas de barbear, bisturi, agulhas, escalpes, ampolas de vidro, lâminas e outros assemelhados provenientes de serviços de Saúde.

Os resíduos de saúde apresentam três dimensões principais de risco: para quem manipula os resíduos, para o aumento das taxas de infecção hospitalar, se não forem gerenciados, e impactando o meio ambiente, se não forem bem destinados. O maior problema dos RSSS é seu potencial de risco. O risco é definido como a medida da probabilidade e da sua severidade decorrerem efeitos adversos de uma ação particular. O risco ambiental pode ser classificado de acordo com o tipo de atividade, englobando as dimensões de exposição instantâneas, crônica, probabilidade de ocorrência, severidade, reversibilidade, visibilidade, duração e ubiqüidade de seus efeitos (NAIME, 2004).

Conforme vários autores (RAMALHO, NAIME e NAIME, 2007 e NAIME, 2004) citam, os impactos ambientais causados pelo gerenciamento inadequado dos resíduos hospitalares podem atingir 
grandes proporções, epidemias ou mesmo endemias devido a contaminações do lençol freático pelos diversos tipos de resíduos dos serviços de saúde.

Os resíduos gerados pelos serviços de saúde podem conter microrganismos que são fontes de contaminação da saúde humana e ambiental, já que determinados organismos podem sobreviver por tempo considerável no lixo hospitalar. De acordo com Hinrischsen (2004) o tempo de sobrevivência do agente patogênico Entamoeba histolytica (desenteria amebiana) é de 8 a 12 dias, Mycobacterium tuberculosis (bacilo da tuberculose) pode sobreviver até 180 dias, e o nematódio Ascaris lumbricóides (Ascaridíase) sobrevive até 2.500 dias.

Especificamente, no que se refere aos RSSS, verifica-se ainda uma ausência de orientação técnico-científica consolidada, onde as discussões sobre os riscos potenciais, bem como as possibilidades de manejo e tratamento dessa categoria de resíduos, são entrecortadas por estereótipos e escassa disponibilidade de dados e informações com rigor científico, especialmente no que tange à forma de tratamento e destinação final (MACHADO, 2004).

Conforme Naime (2004), é necessário um estudo de caracterização, como a pesagem e a análise dos resíduos em cada estabelecimento e em cada período do ano, para se determinar a correta natureza dos resíduos dos serviços de saúde em cada estabelecimento.

O objetivo do presente trabalho é realizar um levantamento gravimétrico de resíduos recicláveis, resíduos não-recicláveis, resíduos biológicos e secreções gerados e descartados em procedimentos cirúrgicos no bloco cirúrgico de um hospital da região metropolitana de Porto Alegre para identificar desperdícios de materiais que eventualmente possam ser otimizados e excesso de geração de resíduos ou procedimentos inadequados. O trabalho foi realizado comparando a quantidade de resíduos biológicos, recicláveis, não-recicláveis e secreções produzidas em 6 procedimentos cirúrgicos freqüentes da instituição hospitalar, sendo 2 considerados de grande porte, 2 de porte médio e 2 de porte pequeno.

Comparando os resíduos gerados por procedimentos cirúrgicos de portes diferentes, a administração do hospital objeto da pesquisa vai poder avaliar a quantidade de resíduos brutos gerados, os impactos ambientais causados e pela eventual diferença nas médias obtidas vai procurar determinar a existência de desperdícios, a geração de excessos de resíduos ou procedimentos operacionais inadequados.

Resíduos sólidos de serviços de saúde são todos os resíduos gerados por estabelecimentos prestadores de serviços de saúde: hospitais, clínicas médicas e odontológicas, laboratórios de análises clínicas e postos de coleta, ambulatórios médicos, farmácias e drogarias, unidades municipais de saúde (postos da rede pública), clínicas veterinárias e instituições de ensino e pesquisa médica, relacionados tanto à população humana quanto à veterinária (COELHO, 2000; NÓBREGA et al., 2002). Dentro de uma instituição hospitalar, várias são as dimensões da questão ambiental, todas elas muito importantes, complexas e dignas de tratamento sério e sistêmico em seu conjunto. No entanto é inegável a emergência e a criticidade da gestão dos resíduos hospitalares ou resíduos dos serviços de saúde.

Entre as principais causas do crescimento da geração de resíduos dos serviços de saúde, está o contínuo incremento da complexidade dos procedimentos e a universalização do sistema (SANCHES, 1995). A medicina somente tornou-se uma prática hospitalar no século XVIII. O hospital até essa época era um local de acúmulo de doentes, ou seja, era um foco de doenças que causavam efeitos negativos à saúde e ao meio ambiente. Alguns estudos começam a ser desenvolvidos com o objetivo de avaliar as funcionalidades do hospital sob o ponto de vista de seus impactos, inicia-se a preocupação com o hospital em relação ao espaço que está situado e sua ligação com a cidade. O hospital precisa ser localizado em um espaço que não seja favorável à propagação de "miasmas, ar poluído e água suja" (FOUCAULT, apud DIAS, 2004).

Ampliando as discussões sobre os riscos associados aos resíduos do serviço da saúde, trabalhos científicos confirmam o reconhecimento dos riscos desses resíduos pela sobrevivência de agentes dotados de elevada resistência às condições ambientais. De forma a destacar outras características peculiares de agentes biológicos na presença de resíduos do serviço de saúde, recente estudo realizado pela Associação de Pesquisa Japonesa, apresentando o estágio ambiental de patógenos nesses resíduos, foi demonstrado a possibilidade de sobrevivência da dose infectante do vírus da Hepatite B ou C, durante uma semana, em uma gota de sangue retirada de uma agulha hipodérmica (PRUSS et al., 1999).

Deste cenário de avaliação de risco envolvendo algumas frações específicas dos RSSS, há consideração de outros possíveis mecanismos de sobrevivência de patógenos na massa desses resíduos, como por exemplo, os materiais biológicos contaminados que podem se constituir em 
importantes veículos para os microrganismos produtores de doenças, quando não devidamente manuseados ou tratados adequadamente.

No contexto nacional, as opiniões divergentes sobre o risco de periculosidade dos RSSS, na sua maioria, são direcionadas à ausência de fatos que comprovem que esses resíduos causem doenças nas pessoas que desenvolvem atividades nos serviços de saúde (SILVA, 2001). Por outro lado, a literatura relata a importância de riscos infecciosos associados aos resíduos sólidos do serviço da saúde, principalmente aos materiais perfurocortantes, como principal perigo à saúde ocupacional (FERREIRA, 1995)

Estes dados estimulam a discussão sobre a forma de gestão dos resíduos hospitalares e dos serviços de saúde em geral. Os interesses econômicos práticos aumentam ainda mais esta discussão. Bencko et al. (2003) discutindo a situação do Hospital Geral de Praga, instituição fundada em 1790, demonstram que o custo para disposição de resíduos comuns é de 75 euros, enquanto o custo para tratamento e disposição dos resíduos infectantes de natureza hospitalar atinge 260 euros/ tonelada. Esses autores asseguram que essa diferença é significativa no orçamento da instituição e justifica o estabelecimento de desafios futuros para aprimorar os tratamentos na instituição.

Conforme Miyazaki et al. (2005), no Japão, os resíduos infecciosos não são coletados e transportados pelo governo municipal, portanto, as instituições médicas são responsáveis pela despesa, e devem contratar empresa específica autorizada para o tratamento desses resíduos. O custo da coleta e transporte está transformando-se em matéria séria. O preço é determinado conforme a quantidade, distância e frequencia do transporte e preço do recipiente. O padrão dos recipientes foi regulado em 1995. Os preços para tratamento desses resíduos estão estimados entre yen 100-150/ $\mathrm{kg}$ ou seja, variam de 900,00 a 1.300,00 dólares/ tonelada. No Japão, Miyazaki et al. (2005) citam que as práticas de gestão de resíduos são feitas de acordo com a "Waste Disposal Law" de 1970, sendo que a gestão de resíduos infecciosos foi regulamentada em 1992, sendo revisada em 2004.

O Hospital Geral Universitário (GUH) de Praga, tem sua própria diretriz para o gerenciamento de todos os tipos de resíduos. Uma pessoa é nomeada como responsável para o tratamento dos resíduos para controlar os mesmos em cada área de trabalho, clínica e instituto. A gerência operacional é responsável por todos os resíduos do hospital. A diretriz orientadora lista regras básicas para segurança, medidas pessoais de pro- teção e emergências em caso de acidentes, baseada na lei de proteção à saúde pública. No GUH a idéia predominante sugere abandonar a filosofia anterior de que todos os resíduos hospitalares devem ser incinerados. Deve haver promoção de um sistema para separar material contaminado do não contaminado. Isto permitirá a reciclagem. Ainda Bencko et. al (2003) cita a recomendação crucial da Conferência de Copenhagen em maio de 1991: separar resíduos perigosos dos não perigosos que não requerem nenhuma manipulação ou eliminação especial.

No Brasil, um hospital com 700 leitos produz aproximadamente 20 tonelada/mês de resíduos biológicos. Considerando os valores praticados no nosso mercado, que podem variar muito de uma região para outra, mas que em Porto Alegre está em média $\mathrm{R} \$ 1000,00 /$ tonelada; a estimativa é de que os hospitais de grande porte estejam gastando R $\$$ $20.000,00 /$ mês com o tratamento desses resíduos.

No caso brasileiro, embora algumas ações estejam em curso para alterar a forma de visão e gestão atual, observa-se que a maioria dos resíduos ainda é considerada como perigosa (infectante ou especial). Essa visão tem como premissa que todo resíduo originado do sistema de saúde esteja contaminado, gerando um preconceito que induz a uma negligência nas políticas de gestão (NAIME et al, 2004). A ideia predominante sugere abandonar a filosofia anterior de que todos os resíduos hospitalares devem ser tratados como infecciosos. Deve haver promoção de um sistema para separar material contaminado do não contaminado. A recomendação crucial da Conferência de Copenhagen em maio de 1991 é separar resíduos perigosos dos não perigosos que não requerem nenhuma manipulação e eliminação especial (BENCKO et al., 2003).

No Brasil, há mais de 30 mil unidades de saúde produzindo esses resíduos, e na maioria das cidades, a questão do manuseio e da disposição final não está resolvida, e acrescenta-se que algumas unidades de saúde desconhecem a quantidade e a composição dos resíduos que produzem (FERREIRA, 1995).

Em serviços de saúde, especificamente, o grande aumento de demanda verificado desde a implantação do Sistema Único de Saúde (SUS) no Brasil, que não foi acompanhado por dotações orçamentárias do mesmo porte, e que portanto, geraram uma defasagem na administração global do sistema, e a crescente utilização de materiais descartáveis como forma de controle mais eficiente das infecções e outros fatores associados, tem contribuído decisivamente para o aumento 
da geração de resíduos.

A população brasileira tem se concentrado, cada vez mais nas áreas urbanas, e a expectativa de vida média do brasileiro vem crescendo. Estes fatores também se somam aos anteriores nas justificativas para o aumento da geração de resíduos dos serviços de saúde. Também influem na natureza e na quantidade dos resíduos produzidos fatores culturais regionais e procedimentos adotados, que dependem de fatores sazonais e até do tipo de alimentação adotado (FORMAGGIA, 1995).

Para Petranovich (1991) o volume dos resíduos dos serviços de saúde tem crescido a uma taxa de $3 \%$ por ano, devido ao fato de que o uso de descartáveis aumentou de $5 \%$ para $8 \%$ ao ano, em função das doenças infecto-contagiosas e da busca de melhores condições nos serviços de saúde. Atualmente, as resoluções do CONAMA anteriores à data da publicação da Resolução 358 de 29 de abril de 2005 foram revogadas. Essa nova resolução do CONAMA guarda extrema coerência e sintonia com a Resolução 306/2004 da Agência Nacional de Vigilância Sanitária (ANVISA, 2004). Para Naime 2005a:

a falta de informações sobre o assunto é um dos principais motivos para a ausência de projetos bem sustentados que determinem melhorias no setor. Particularmente os resíduos dos serviços de saúde merecem atenção especial em suas fases de separação, acondicionamento, armazenamento, coleta, transporte, tratamento e disposição final.

As consequências da falta de informações e indefinições são observadas pela carência de modelos de resíduos do serviço da saúde, pois uma parte considerável das organizações hospitalares desconhece os procedimentos básicos no manejo dos resíduos. Por outro lado, cabe destacar que a concepção dos tratamentos de resíduos pode avançar muito. Autores como Andrade (1999) apresentam os resultados encontrados para a composição gravimétrica dos resíduos produzidos por estabelecimentos prestadores de serviços de saúde no município de São Carlos, em São Paulo, demonstrando que há predominância de materiais potencialmente reaproveitáveis entre esses resíduos. Levando esse fato em consideração, que muitos resíduos de saúde não são contaminados, e apropriando a idéia de gestão avançada de resíduos, muitas ações podem ser aprimoradas nessa questão.

É importante salientar a existência de estudos sobre a presença de patógenos oportunistas nos líquidos percolados da disposição final de
RSSS, que revelam microrganismos indicadores de contaminação ambiental como a $P$. aeruginosa, $S$. aureus, E. fecais e $C$. perfringens (BIDONE et al., 2000). Essas evidências, somadas às outras, facilitam a elaboração de um planejamento e implantação de ações sistêmicas. Atualmente existem legislações federais extremamente rigorosas com a responsabilidade do gerador sobre os resíduos gerados (NAIME, 2005b).

Tema transversal e multidisciplinar para várias áreas, desde a medicina até a administração hospitalar, a questão do gerenciamento e destinação final adequados dos resíduos sólidos dos serviços de saúde tem sua mais recente normatização fixada pela Agência Nacional de Vigilância Sanitária (ANVISA). Este procedimento foi realizado através de resolução conjunta com o CONAMA, com o título de "Regulamento Técnico para o Gerenciamento de Resíduos de Serviços de Saúde", sob o número de Resolução - RDC 306 do órgão.

Este Regulamento apresenta no capítulo III Gerenciamento dos Resíduos de Serviços de Saúde o seguinte parágrafo:

O gerenciamento dos RSS constitui-se em um conjunto de procedimentos de gestão, planejados e implementados a partir de bases científicas e técnicas, normativas e legais, com o objetivo de minimizar a produção de resíduos e proporcionar aos resíduos gerados, um encaminhamento seguro, de forma eficiente, visando a proteção dos trabalhadores, a preservação da saúde pública, dos recursos naturais e do meio ambiente. (RDC 306/2004 - ANVISA).

A partir dessas orientações, todo gerador deve elaborar um Plano de Gerenciamento de Resíduos de Serviços de Saúde (PGRSS), conforme as características dos resíduos gerados e na classificação especificada na Resolução. Esse Plano engloba o manejo nas etapas de segregação, acondicionamento, identificação, transporte interno, armazenamento temporário, tratamento, armazenamento externo, coleta e transporte externo e disposição final. Esse Plano será documento obrigatório para a solicitação de licenciamento ambiental das empresas que geram resíduos de serviços de saúde.

\section{MATERIAIS E MÉTODOS}

O local de estudo foi a Unidade do Bloco Cirúrgico de um hospital na região metropolitana 
de Porto Alegre, que possui uma média diária de 55 procedimentos. Essa unidade é composta por doze salas de procedimentos, uma sala de preparo (infantil e adulto) e uma sala de recuperação. A Unidade de Bloco Cirúrgico possui um corredor central, ao longo deste encontram-se as salas de cirurgias que dão acesso a um corredor secundário, também chamado de corredor ímpar, por onde circulam os resíduos retirados das salas cirúrgicas. $\mathrm{Na}$ Unidade do Bloco Cirúrgico foram avaliados seis tipos de procedimentos. Foram pesados os resíduos provenientes destes seis tipos diferentes de cirurgias em quatro categorias: resíduos biológicos, recicláveis, não-recicláveis e secreções, totalizando 30 amostras, sendo 5 de cada procedimento considerado, com 4 pesagens em cada. Para auxiliar o levantamento gravimétrico dos procedimentos foi elaborado um quadro de acompanhamento para cada procedimento.

Após o término do procedimento cirúrgico, os auxiliares de higienização realizam a limpeza, a desinfecção e a retirada dos resíduos das salas cirúrgicas que, por sua vez, já saem devidamente segregados. Os resíduos que foram pesados na UBC são acondicionados em sacos de cores diferenciadas que caracterizam a sua periculosidade. Esses sacos possuem espessura e tamanho apropriados, de acordo com a composição e o peso do resíduo, resistência e impermeabilidade.

Os resíduos do GRUPO A são acondicionados em sacos plásticos na cor branca e os do GRUPO D em sacos plásticos nas cores: verde para materiais recicláveis e, preto para materiais não-recicláveis, que por sua vez, são fechados com dois nós bem apertados antes da sua pesagem. São citados somente os resíduos do GRUPO A e do GRUPO D, uma vez que os procedimentos citados descartam resíduos pertencentes a esses grupos.

Os sacos foram encaminhados para a sala de expurgo, localizada no corredor ímpar, onde a balança foi instalada. Após a chegada dos resíduos ocorreu a pesagem e seus dados anotados no quadro de acompanhamento. Para efetuar a gravimetria utilizou-se EPIs como luvas de borracha e óculos de proteção priorizando a segurança para que não ocorra o risco de contaminação. As pesagens foram realizadas com uma balança digital de plataforma da marca Filizola, Modelo ID-M 150/4 com capacidade máxima de $150 \mathrm{~kg}$ e variação de $50 \mathrm{~g}$.

As secreções foram retiradas das salas cirúrgicas em recipientes de vidro ou em baldes de aço inoxidável que foram previamente pesados para posteriormente desconsiderarmos os seus valores, uma vez que, eles serão higienizados e reutilizados. As pesagens foram realizadas com a mesma balança utilizada para as pesagens dos outros tipos de resíduos. Partindo dos dados amostrais coletados foram analisados posteriormente $o$ cálculo dos pesos totais para cada procedimento cirúrgico e ainda as médias por tipo de resíduos. Foram realizadas análises estatísticas com teste "t" de Student,com nível de relevância de até 5\%, entre as 10 amostras de cirúrgias consideradas de grande porte com as 10 amostras de cirúgias de médio porte e as 10 amostras de cirúrgias de médio porte. Assim a instituição hospitalar pesquisa pela primeira vez dados fundamentais para o exercício de um planejamento eficiente na produção, geração, gerenciamento e destinação de resíduos sólidos dos serviços de saúde na área cirúrgica. As intepretações dos dados objetivam trazer informações para a melhoria dos procedimentos e rotinas visando a otimização na geração de resíduos e nas ações de proteção ambiental.

\section{ANÁLISE DOS RESULTADOS}

Foram realizadas cinco pesagens gravimétricas para cada um dos seis procedimentos cirúrgicos considerados, incluindo Revascularização do Miocárdio, Setorectomia, Colecistectomia Videolaparoscópica, Tireoidectomia Total, Exerese e Sutura de lesão e Microcirurgia Endonasal. As pesagens consideraram os ítens de resíduos biológicos, resíduos recicláveis, resíduos não-recicláveis, secreções e recipientes. As pesagens dos procedimentos foram realizadas entre 18 de dezembro de 2008 e 31 de agosto de 2009. Os quadros 1, 2, $3,4,5$ e 6 abaixo apresentam os resultados para cada procedimento, incluindo uma coluna com o total da pesagem encontrada.

Diante dos indicadores obtidos com a pesquisa torna-se importante ressaltar que é possível classificar os resíduos biológicos ou infectantes como aqueles que possuem agentes biológicos, ou estão contaminados por agentes biológicos, e geram riscos potenciais à saúde dos funcionários ou à da população. Resíduos recicláveis são todos aqueles que podem ser novamente reutilizados através da reciclagem, e resíduos não-recicláveis não podem ser reutilizados devido à falta de tecnologia para a sua reciclagem ou simplesmente por serem compostos por materiais que não permitem a sua reciclagem.

A primeira comparação foram entre as pesagens de dois tipos de cirúrgicas de grande porte e dois tipos de médio porte nas quatro categorias já descritas e constantes do eixo $\mathrm{X}$ da Figura 1 que mostra os resultados. 
Quadro 1: Dados do procedimento de Revascularização do Miocárdio.

\begin{tabular}{|c|c|c|c|c|c|c|c|}
\hline N & Data & $\begin{array}{c}\text { Res. Biol. } \\
(\mathrm{kg})\end{array}$ & $\begin{array}{c}\text { Res. Rec. } \\
(\mathrm{kg})\end{array}$ & $\begin{array}{c}\text { Res. Não } \\
\text { Rec. }(\mathrm{kg})\end{array}$ & $\begin{array}{c}\text { Secreção } \\
(\mathrm{kg})\end{array}$ & $\begin{array}{c}\text { Recip. } \\
(\mathrm{kg})\end{array}$ & $\begin{array}{c}\text { Peso Total } \\
(\mathrm{kg})\end{array}$ \\
\hline 1 & $15 / 01 / 09$ & 10,25 & 2,35 & 0,25 & 3,90 & 2,90 & 19,65 \\
\hline 2 & $15 / 01 / 09$ & 9,65 & 1,45 & 0,15 & 3,85 & 2,80 & 17,90 \\
\hline 3 & $19 / 01 / 09$ & 9,20 & 2,40 & 0,40 & 3,80 & 2,80 & 18,60 \\
\hline 4 & $19 / 01 / 09$ & 10,75 & 2,45 & 0,30 & 4,45 & 2,80 & 19,75 \\
\hline 5 & $20 / 01 / 09$ & 9,30 & 2,20 & 0,55 & 4,55 & 2,80 & 20,30 \\
\hline Médias & - & $\mathbf{9 , 8 3}$ & $\mathbf{2 , 1 7}$ & $\mathbf{0 , 3 3}$ & $\mathbf{4 , 1 1}$ & $\mathbf{2 , 8 2}$ & $\mathbf{1 9 , 2 4}$ \\
\hline
\end{tabular}

Fonte: Dados dos autores (2009).

Quadro 2: Dados do procedimento de Setorectomia

\begin{tabular}{|c|c|c|c|c|c|c|c|}
\hline $\mathrm{N}$ & Data & $\begin{array}{l}\text { Res. Biol. } \\
(\mathrm{kg})\end{array}$ & $\begin{array}{l}\text { Res. Rec. } \\
(\mathrm{kg})\end{array}$ & $\begin{array}{l}\text { Res. Não } \\
\text { Rec. (kg) }\end{array}$ & $\begin{array}{l}\text { Secreção } \\
(\mathrm{kg})\end{array}$ & Recip. (kg) & $\begin{array}{l}\text { Peso Total } \\
(\mathrm{kg})\end{array}$ \\
\hline 1 & $26 / 08 / 09$ & 0,550 & 0,450 & 0,150 & 0 & 0 & 1,150 \\
\hline 2 & $26 / 08 / 09$ & 0,550 & 0,250 & 0,100 & 0 & 0 & 0,900 \\
\hline 3 & $2708 / 09$ & 0,450 & 0,300 & 0,050 & 0 & 0 & 0,800 \\
\hline 4 & $31 / 08 / 09$ & 0,400 & 0,450 & 0,150 & 0 & 0 & 1,00 \\
\hline 5 & $31 / 08 / 09$ & 0,550 & 0,400 & 0,100 & 0 & 0 & 1,05 \\
\hline Médias & - & 0,50 & 0,37 & 0,11 & 0 & 0 & 0,980 \\
\hline
\end{tabular}

Fonte: Dados dos autores (2009).

Quadro 3: Dados do procedimento de Colecistectomia Videolaparoscópica.

\begin{tabular}{|c|c|c|c|c|c|c|c|}
\hline $\mathbf{N}$ & Data & $\begin{array}{c}\text { Res. Biol. } \\
(\mathrm{kg})\end{array}$ & $\begin{array}{c}\text { Res. } \\
\text { Rec. }(\mathrm{kg})\end{array}$ & $\begin{array}{c}\text { Res. Não } \\
\text { Rec. }(\mathrm{kg})\end{array}$ & $\begin{array}{c}\text { Secreção } \\
(\mathrm{kg})\end{array}$ & $\begin{array}{c}\text { Recip. } \\
(\mathrm{kg})\end{array}$ & $\begin{array}{c}\text { Peso Total } \\
(\mathrm{kg})\end{array}$ \\
\hline 1 & $18 / 12 / 08$ & 1,25 & 0,80 & 0,20 & 0,250 & 1,55 & 5,60 \\
\hline 2 & $12 / 01 / 09$ & 0,70 & 0,20 & 0,10 & 0,050 & 1,55 & 4,15 \\
\hline 3 & $12 / 01 / 09$ & 1,10 & 0,45 & 0,20 & 0,750 & 1,55 & 5,60 \\
\hline 4 & $12 / 01 / 09$ & 1,05 & 0,75 & 0,10 & 0,100 & 1,55 & 5,10 \\
\hline 5 & $14 / 01 / 09$ & 1,25 & 0,25 & 0,05 & 0,850 & 1,55 & 5,50 \\
\hline Médias & - & $\mathbf{1 , 0 7}$ & $\mathbf{0 , 4 9}$ & $\mathbf{0 , 1 3}$ & $\mathbf{0 , 7 0}$ & $\mathbf{1 , 5 5}$ & $\mathbf{5 , 1 9}$ \\
\hline
\end{tabular}

Fonte: Dados dos autores (2008/2009).

Quadro 4: Dados do procedimento de Tireoidectomia Total.

\begin{tabular}{|c|c|c|c|c|c|c|c|}
\hline $\mathbf{N}$ & Data & $\begin{array}{c}\text { Res. Biol. } \\
(\mathrm{kg})\end{array}$ & $\begin{array}{c}\text { Res. Rec. } \\
(\mathrm{kg})\end{array}$ & $\begin{array}{c}\text { Res. Não } \\
\text { Rec. }(\mathrm{kg})\end{array}$ & $\begin{array}{c}\text { Secreção } \\
(\mathrm{kg})\end{array}$ & $\begin{array}{c}\text { Recip. } \\
(\mathrm{kg})\end{array}$ & $\begin{array}{c}\text { Peso Total } \\
(\mathrm{kg})\end{array}$ \\
\hline 1 & $06 / 08 / 09$ & 2,500 & 0,550 & 0,150 & 0 & 0 & 3,200 \\
\hline 2 & $06 / 08 / 09$ & 2,300 & 0,450 & 0,150 & 0 & 0 & 2,900 \\
\hline 3 & $24 / 08 / 09$ & 1,600 & 0,600 & 0,100 & 0 & 0 & 2,300 \\
\hline 4 & $27 / 08 / 09$ & 0,750 & 0,300 & 0,250 & 0 & 0 & 1,300 \\
\hline 5 & $27 / 08 / 09$ & 2,100 & 0,450 & 0,150 & 0 & 0 & 2,700 \\
\hline Médias & - & $\mathbf{1 , 8 5}$ & $\mathbf{2 , 3 5}$ & $\mathbf{0 , 1 6}$ & $\mathbf{0}$ & $\mathbf{0}$ & $\mathbf{2 , 4 8 0}$ \\
\hline
\end{tabular}

Fonte: Dados dos autores (2009). 
Quadro 5: Dados do procedimento de Exerese e Sutura de lesão.

\begin{tabular}{|c|c|c|c|c|c|c|c|}
\hline $\mathrm{N}$ & Data & $\begin{array}{c}\text { Res. Biol. } \\
(\mathrm{kg})\end{array}$ & $\begin{array}{c}\text { Res. Rec. } \\
(\mathrm{kg})\end{array}$ & $\begin{array}{c}\text { Res. Não } \\
\text { Rec. }(\mathrm{kg})\end{array}$ & $\begin{array}{c}\text { Secreção } \\
(\mathrm{kg})\end{array}$ & $\begin{array}{c}\text { Recip. } \\
(\mathrm{kg})\end{array}$ & $\begin{array}{c}\text { Peso Total } \\
(\mathrm{kg})\end{array}$ \\
\hline 1 & $19 / 08 / 09$ & 1,050 & 0,150 & 0,100 & 0,050 & 1,550 & 1,350 \\
\hline 2 & $20 / 08 / 09$ & 0,350 & 0,050 & 0,100 & 0 & 0 & 0,500 \\
\hline 3 & $20 / 08 / 09$ & 0,500 & 0,150 & 0,100 & 0 & 0 & 0,750 \\
\hline 4 & $24 / 08 / 09$ & 0,300 & 0,200 & 0,100 & 0 & 0 & 0,600 \\
\hline 5 & $25 / 08 / 09$ & 1,650 & 0,750 & 0,100 & 0 & 0 & 2,500 \\
\hline Médias & - & $\mathbf{0 , 5 7}$ & $\mathbf{0 , 2 6}$ & $\mathbf{0 , 0 2}$ & $\mathbf{0 , 0 1}$ & $\mathbf{0 , 3 1}$ & $\mathbf{1 , 1 4}$ \\
\hline
\end{tabular}

Fonte: Dados dos autores (2009).

Quadro 6: Dados do procedimento de Microcirurgia Endonasal.

\begin{tabular}{|c|c|c|c|c|c|c|c|}
\hline $\mathbf{N}$ & Data & $\begin{array}{c}\text { Res. Biol. } \\
(\mathrm{kg})\end{array}$ & $\begin{array}{c}\text { Res. Rec. } \\
(\mathrm{kg})\end{array}$ & $\begin{array}{c}\text { Res. Não } \\
\text { Rec. }(\mathrm{kg})\end{array}$ & $\begin{array}{c}\text { Secreção } \\
(\mathrm{kg})\end{array}$ & $\begin{array}{c}\text { Recip. } \\
(\mathrm{kg})\end{array}$ & $\begin{array}{c}\text { Peso Total } \\
(\mathrm{kg})\end{array}$ \\
\hline 1 & $26 / 08 / 09$ & 0,600 & 0,650 & 0,100 & 0,250 & 1,550 & 1,600 \\
\hline 2 & $26 / 08 / 09$ & 0,550 & 0,500 & 0,100 & 0,100 & 1,550 & 1,250 \\
\hline 3 & $26 / 08 / 09$ & 0,500 & 0,600 & 0,150 & 0,200 & 1,550 & 1,450 \\
\hline 4 & $27 / 08 / 09$ & 0,600 & 0,650 & 0,050 & 0,300 & 1,550 & 1,600 \\
\hline 5 & $28 / 08 / 09$ & 0,550 & 0,550 & 0,100 & 0,250 & 1,550 & 1,450 \\
\hline Médias & - & $\mathbf{0 , 5 6}$ & $\mathbf{0 , 5 9}$ & $\mathbf{0 , 1 0}$ & $\mathbf{0 , 2 2}$ & $\mathbf{1 , 5 5}$ & $\mathbf{1 , 4 7}$ \\
\hline
\end{tabular}

Fonte: Dados dos autores (2009).

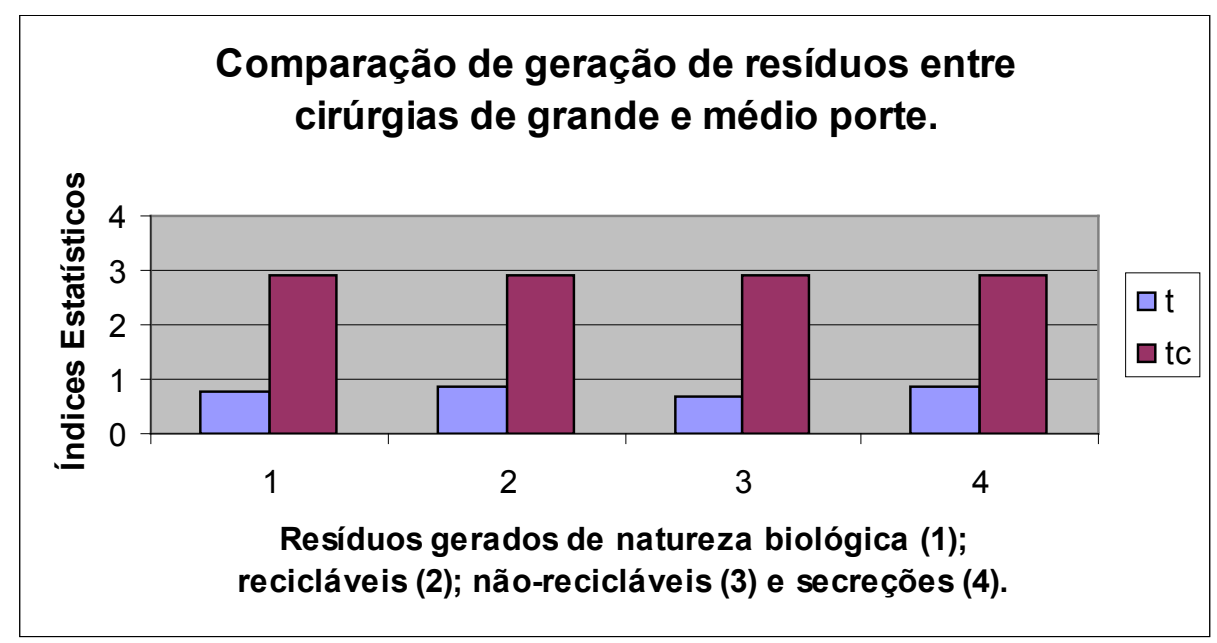

Figura 1: Comparação estatística entre cirúrgias de grande e médio porte quanto a geração de resíduos biológicos, recicláveis, não-recicláveis e secreções.

Comparando as cirúrgias de grande e médio porte não existe variação significativa ou relevante de nenhum dos tipos de resíduos comparados. As populações de pesagens de resíduos de natureza biológica, reciclável, não-recicláveis e secreções constituem populações únicas nas quantidades geradas. Este fato já foi uma novidade pois o hospital acreditava que cirúrgias de maior porte produziam maiores quantidades de resíduos e isto estatisticamente não é verdadeiro. Sempre que o "t" calculado for menor que o "tc" ou "t" crítico, a interpretação estatística é que se tratam de populações equivalentes ou que não existem diferenças relevantes entre as amostragens comparadas. 


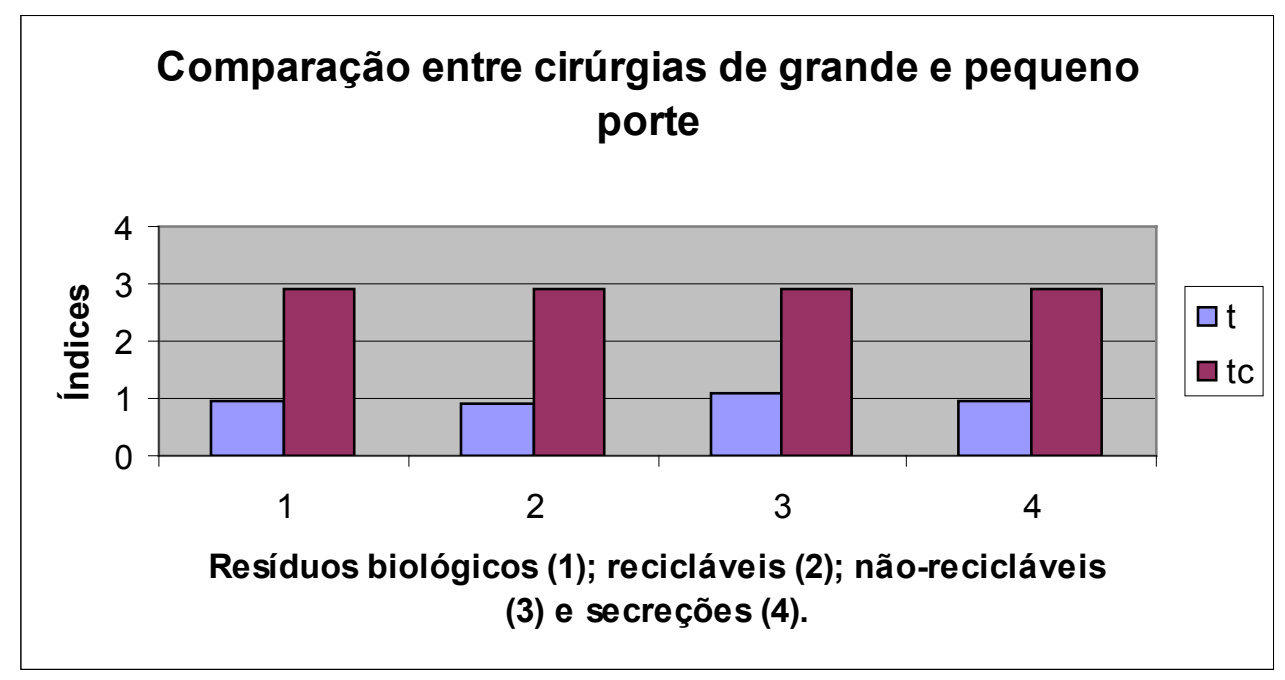

Figura 2: Tratamento estatístico dos dados entre as 4 variáveis analisadas, entre procedimentos cirúrgicos de grande e pequeno porte.

As secreções orgânicas é uma forma em que as células dos corpos vivos encontram para liberar substâncias para o meio externo. As secreções e os materiais contaminados por ela como gazes, algodões, ataduras e outros também são considerados resíduos biológicos. Neste estudo foi considerado como secreção todo o líquido aspirado durante o procedimento cirúrgico, que por sua vez, caracteriza-se por sangue, gordura, linfa e plasma.

$\mathrm{Na}$ comparação estatística entre as cirúrgias de grande porte com as de pequeno porte, a surpresa foi ainda maior, na medida em que as comparações de pesagens tratadas estatisticamente com o teste "t" de Student, demonstraram que não existem diferenças relevantes entre as populações. Tanto os resíduos de natureza biológica, quanto os recicláveis, não-recicláveis e secreções são equivalentes.

Os resultados do tratamento estão apresen- tados na Figura 2 a seguir. A estatística desmistifica uma idéia generalizada de que cirúrgias de grande porte eram mais expressivas na geração de resíduos do que cirúrgias de pequeno porte. Há uma equivalência nas 4 categorias de resíduos mensuradas por gravimetria.

Isto significou imediatamente para a direção do hospital que todas as cirúrgias devem ser tratadas da mesma forma quanto a geração de resíduos e que não se deve negligenciar um procedimento pelo mesmo ser considerado de pequeno porte. Estas constatações podem parecer simplórias, mas na verdade é desconhecida iniciativa semelhante de instituição hospitalar de buscar obter conhecimento detalhado sobre o tema para orientar suas políticas de procedimentos, normas e gestão. E até mesmo durante a execução do trabalho os resultados encontrados causavam surpresa e podiam ser

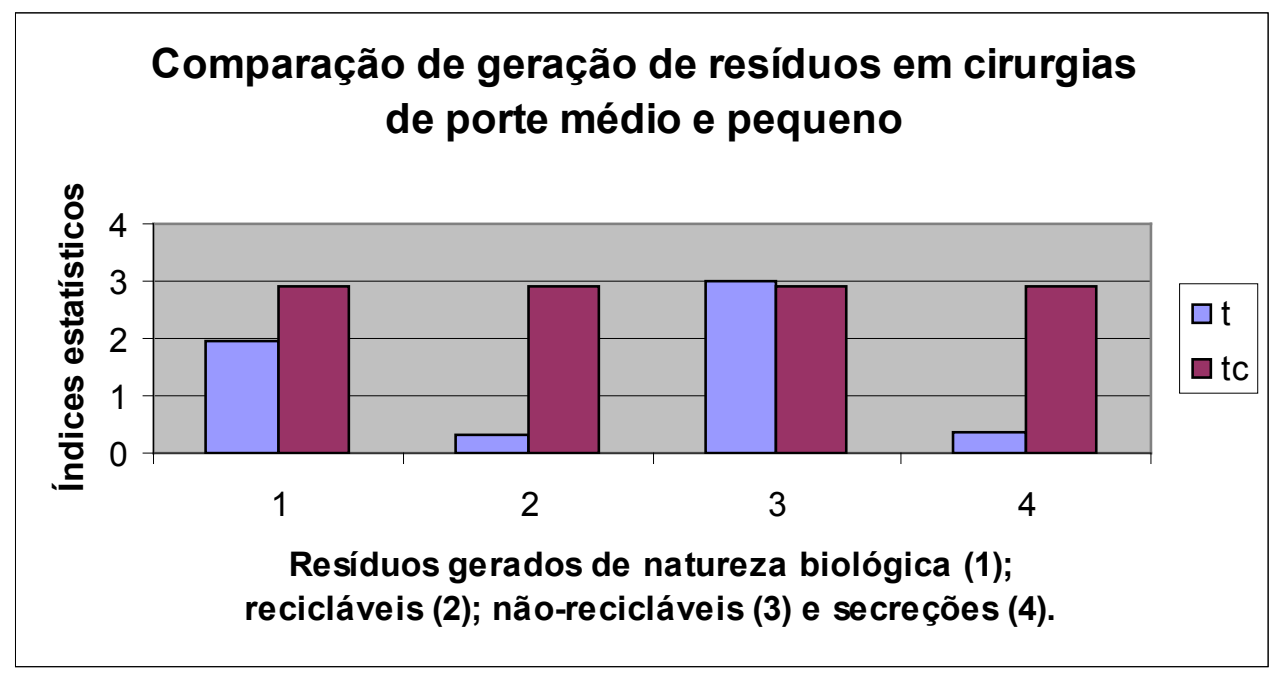

Figura 3: Resultados das comparações estatísticas entre os resíduos sólidos de natureza biológica, recicláveis, não-recicláveis e secreções de procedimentos cirúrgicos considerados de médio e pequeno porte. 
observados que os resultados gravimétricos dos 4 itens selecionados eram muito diferenciados entre si e não guardavam relação direta com qualquer expectativa prévia.

Apenas na comparação entre procedimentos cirúrgicos de porte médio com cirúrgias consideradas de pequeno porte foi encontrada alteração significativa no item não recicláveis, atestando que as duas cirurgias não produzem quantidades equivalentes. Nos demais itens não houveram variações significativas de resíduos sólidos de serviços de saúde produzidos. Os resultados se encontram na Figura 3.

Os resultados encontrados, mais do que interpretações pontuais, permitem a formulação de procedimentos e normas satisfatórias para minimização na geração de resíduos dos serviços de saúde além de otimização das práticas de gestão e proteção ambiental. Apenas nos resíduos não recicláveis o valor de " $t$ " demonstra que podem haver diferenças relevantes entre as amostras representativas das comparações consideradas.

\section{CONSIDERAÇÕES FINAIS}

É crescente a preocupação com a geração de resíduos sólidos impactantes que podem vir a gerar alterações no meio ambiente e na qualidade de vida das populações. Nos resíduos sólidos dos serviços de saúde, há uma parte que são resíduos recicláveis e devem ter esta destinação. Mas há uma parte que deve receber tratamento adequado com a destinação para (ARIPs) ou tratamento prévio com autoclavagem e destinação final em ARIPs. Este trabalho demonstra que não existem diferenças relevantes entre as tipologias de intervenções cirúrgicas comparadas.

A colecistectomia videolaparoscópica é uma intervenção cirúrgica bastante comum, de elevada ocorrência diária no bloco cirúrgico devido a sua média complexidade e seu tempo de execução ser relativamente curto. E o procedimento de revascularizaçao do miocárdio com CEC é uma intervenção de baixa ocorrência diária devido a sua alta complexidade e seu tempo de duração muito longo.

Realizando a comparação estatística entre a geração de resíduos sólidos de serviços de saúde desses procedimentos cirúrgicos diferenciados conclui-se que a natureza da intervenção influencia a geração dos resíduos sólidos de saúde.

Por fim, é importante destacar que esta pesquisa tem como limite o fato de ter realizado a investigação somente de um bloco cirúrgico envolvendo seis tipos diferentes de procedimentos cirúrgicos. Entretanto, mesmo considerando que a amostragem da pesquisa possa ser ampliada foi possível encontrar evidências para a hipótese proposta neste trabalho. A partir desses indicativos propõe-se como sugestão para investigações futuras ampliar o universo amostral, bem como focar na questão da destinação dos resíduos gerados, uma vez que os riscos ambientais e para a saúde humana são preocupações pertinentes ao manuseio desses resíduos.

\section{REFERÊNCIAS BIBLIOGRÁFICAS}

AGÊNCIA NACIONAL DE VIGILÂNCIA SANITÁRIA (ANVISA). Resolução da Diretoria Colegiada 306 de 7 de dezembro de 2004.

ANDRADE, J. B. L. Determinação da composição gravimétrica dos resíduos de serviços de saúde de diferentes tipos de estabelecimentos geradores. In: Congresso Brasileiro de Engenharia Sanitária e Ambiental, 20., 1999. Rio de Janeiro. Anais... Rio de Janeiro: Associação Brasileira de Engenharia Sanitária e Ambiental, 1999, p. 1666-1672.

ASSOCIAÇÃO MÉDICA BRASILEIRA. Classificação Brasileira Hierarquizada de Procedimentos Médicos. $4^{\circ}$ Ed. p. 15. Disponível em: < www.amb.org.br/instrucoes gerais.pdf $>$ Acesso em: 17 nov. 2008.

BENCKO, V., KAPEK, J.; VINS, O. Hospital Waste Treatment and Disposal in the General University Hospital - Current Situation and Future Challenges. Indoor and Built Environment (12) pg. 99-104, 2003.

BIDONE F. R. A.; POVINELLI, J. Conceitos básicos de resíduos sólidos. São Carlos. EESC/USP. 1999.

CENTRO PAN - AMERICANO DE ENGENHARIA SANITÁRIA E CIÊNCIAS DO AMBIENTE. Guia para manejo interno de resíduos sólidos em estabelecimentos de saúde. Tradução de Carol Castilho Arguello. Brasília: Organização Pan-americana da Saúde, 1997. 17p.

COELHO, H. Manual de gerenciamento de resíduos sólidos de serviços de saúde. Rio de Janeiro: CICT/FIOCRUZ, 2000. 
CONSELHO NACIONAL DO MEIO AMBIENTE (CONAMA). Ministério do Meio Ambiente. Resolução no 5 de 5 /8/93. Brasília. 1993.

CONSELHO NACIONAL DO MEIO AMBIENTE (CONAMA). Ministério do Meio Ambiente. Resolução no 6 de 19 /9/91. Brasília. 1991.

DIAS, M. A. A. Resíduos dos Serviços de Saúde e a Contribuição do Hospital para a Preservação do Meio Ambiente. Revista Academia de Enfermagem, v.2, n. 2, jan/2004. p.21-29.

FERREIRA, J. A. Resíduos Sólidos e Lixo Hospitalar: Uma Discussão Ética. Caderno de Saúde Pública, Rio de janeiro: v.11, n. 2, p. 314-320, Abr-Jun/ 1995.

FORMAGGIA, D. M. E. Resíduos de Serviços de Saúde. In: Gerenciamento de Resíduos sólidos de Serviços de Saúde. São Paulo: CETESB. 1995. p. 3-13.

HINRISCHSEN, S. L. Lixo Hospitalar - Gerenciamento dos Resíduos Sólidos dos Serviços de Saúde, p. 230-245. In: HINRISCHEN. et al. Biossegurança e Controle de Infecções - Risco Sanitário Hospitalar. Rio de Janeiro: Medsi, 2004.

INSTITUTO BRASILEIRO DE GEOGRAFIA E ESTATÍSTICA (IBGE). Pesquisa Nacional de Saneamento Básico - PNSB. 2000. In: REBELATTO, M. F. 2006. Avaliação de Métodos de Desinfecção de Resíduos Infecciosos e de seu Percolado. Florianópolis, Dissertação de Pós-graduação em Engenharia Ambiental - PPGEA do Centro de Tecnológico da Universidade Federal de Santa Catarina, 135p.

Disponível em: <http://www.ibge.gov. $\mathrm{br} /$ home/presidencia/noticias/noticia_visualiza. php?id_noticia $=207>$ Acesso em 01 set. 08 .

INSTITUTO NACIONAL DE CARDIOLOGIA. Ravascularização do Miocárdio. Disponível em: $<$ http://www.incl.rj.saude.gov.br/incl/paginas/ revas.asp $>$. Acessado em: 28 set.08.

MACHADO, N. L.; MORAES, L. R. S.. RSSS: Revisitando as soluções adotadas no Brasil para tratamento e destino final. Revista Engenharia Sanitária e Ambiental, v. 9, n. 1, p. 55-64. 2004

MAGALHÃES, S. Apresentação. In: C. E. VALLE. Qualidade Ambiental. São Paulo: Pioneira, 1995.
MIYAZAKI, M., UNE, H. Infectious waste management in Japan: A revised regulation and a management process in medical institutions. Waste Management 25, pg. 616-621, 2005.

MONREAL, J. Consideraciones sobre el Manejo de Residuos de Hospitalares en América Latina. In: Uma abordagem sobre a gestão de resíduos da saúde, 2004, Londrina-PR. Revista Espaço para a Saúde, Londrina-PR, v. 5, n. 2, p. 17-27.

NAIME, R. Gestão de Resíduos Sólidos: uma abordagem prática. Novo Hamburgo: Feevale, 2004.

NAIME, R. Diagnóstico Ambiental e Sistemas de Gestão Ambiental. Novo Hamburgo: Feevale, 2005a.

Gestão de Resíduos Sólidos. Novo Hamburgo: Feevale, 2005b.

NAIME, R.; SARTOR, I; GARCIA, A. C. Uma abordagem sobre a gestão de resíduos de serviço da saúde. Revista Espaço para a Saúde, Londrina-PR, v. 5 , n. 2, p. 17-27, 2004.

NAIME, R; GARCIA, A. C. A. Percepção ambiental e diretrizes para compreender a questão do meio ambiente. Novo Hamburgo: Feevale, 2004.

NÓBREGA, C.C. et al. Diagnóstico dos resíduos sólidos de serviços de saúde provenientes de hospitais e clínicas médicas do município de João Pessoa - PB. In: SIMPÓSIO ITALO-BRASILEIRO DE ENGENHARIA SANITÁRIA E AMBIENTAL, 6 ., 2002, Vitória. Anais... Vitória. Associação Brasileira de Engenharia Sanitária e Ambiental, 2002. 1 CD- ROM

PETRANOVICH, J. Minimization of Environmental effects from medical waste. Packaging of Health-care Devices and Products. 1991.

PRUSS, A., GIROULT, E., RUSHBROOK, D. Safe Management of Wastes from Health-care Activities. World Health Organization. Geneva. 1999.

RAMALHO, A. H. P.; NAIME, R. H.; NAIME, I. S. 2007. Diagnóstico do Sistema de Gestão dos Resíduos Sólidos do Hospital de Clínicas de Porto Alegre. Estudos Tecnológicos em Engenharia - v. 3, n.1, p.12-36.

RAMALHO, A. P. Plano de Gestão de Resíduos 
Sólidos do Hospital de Clínicas de Porto Alegre. Hospital de Clínicas de Porto Alegre, Porto Alegre. 41p. 2008 (Documento Interno).

\section{REBELATTO, M.F. Avaliação de Métodos de Desinfecção de Resíduo Infeccioso e de seu Perco- 1ado. Florianópolis, Dissertação de Mestrado, Pro- grama de Pós-Graduação em Engenharia Ambiental - PPGEA da Universidade Federal de Santa Cata- rina, 2006.}

SANCHES, P. S. Caracterização dos riscos nos resíduos de sistema de saúde e na comunidade. In: Gerenciamento de Resíduos Sólidos de Serviços de Saúde. CETESB, 1995. p. 33 - 46.

SILVA, A.C.N. Indicadores de Contaminação Ambiental e Diretrizes Técnicas para Disposição Final de Resíduos Sólidos de Serviços de Saúde: Uma Abordagem Multidisciplinar. Dissertação de Mestrado, Brasília: Departamento de Engenharia Civil e Ambiental, Faculdade de Tecnologia, Universidade de Brasília, 2001.

TONETO, M.G.; MOHR, C.C.; LOPES, M. H. I. Das Grandes Incisões Cirúrgicas à Colecistectomia Laparoscópica: Uma Reflexão Sobre o Impacto de Novas Tecnologias. Scientia Medica, Porto Alegre, v. 17, n. 1, p. 31 - 35, Jan./Mar. 2007.

\section{AGRADECIMENTOS}

Ao Programa de Pós graduação em Qualidade Ambiental e aos institutos de Ciências da Saúde (ICS) e de Ciências Tecnológicas e Exatas (ICET) da Universidade FEEVALE e ao Hospital de Clínicas de Porto Alegre pelo apoio na realização deste trabalho. 